\title{
Medición y visibilización de la Economía Social en el País Vasco
}

Dificultades, aproximaciones y propuestas desde el Observatorio Vasco de Economía Social (OVES/GEEB)

\author{
Ignacio BRETOS FERNÁNDEZ \\ Observatorio Vasco de Economía Social (OVES/GEEB), \\ Universidad del País Vasco (UPV/EHU)
}

DOI: $10.1387 /$ reves. 15257

Fecha de entrada: 10/10/2014

Fecha de aceptación: 02/02/2015

Sumario: 1. Introducción. 2. Aproximaciones a la conceptualización, delimitación y medición de la Economía Social. 3. El Observatorio Vasco de Economía Social (OVES/GEEB). 4. Economía Social y crisis económica: resultados en el País Vasco. 5. Dificultades y propuestas desde el OVES/GEEB para la medición de la Economía Social vasca. 6. Conclusiones. 7. Referencias bibliográficas.

\section{Resumen:}

A partir de la experiencia adquirida desde el Observatorio Vasco de Economía Social (OVES/GEEB) en el ámbito de la medición y evaluación de la economía social, en este artículo se identifican las principales dificultades existentes para la obtención de datos estadísticos oficiales sobre las distintas tipologías de entidades de la economía social en el País Vasco, proponiéndose asimismo soluciones para superar dichos obstáculos. Dado el mejor comportamiento de este tipo de entidades frente a la crisis económica en comparación con la empresa tradicional, y asumida la necesidad de que existan estadísticas rigurosas, homogéneas y constantes para la mejor visibilización social e institucional del sector, nuestro interés último radica en contribuir a facilitar la adopción de políticas por parte de las Administraciones Públicas sustentadas en el fomento de la economía social para hacer frente a la crisis económica en el País Vasco.

\section{Palabras clave:}

Economía Social, Crisis Económica, Medición de la Economía Social, Visibilidad de la Economía Social, Políticas Públicas. 


\begin{abstract}
:
Based on the experience acquired from the "Basque Observatory of Social Economy (OVES/GEEB)» in the field of measurement and evaluation of the social economy, this article identifies the main difficulties for obtaining official statistical data on the different types of entities of Social Economy in the Basque Country. Possible solutions are also proposed to overcome these obstacles. Given the better response of this kind of entities to the economic crisis in comparison with traditional firms, and assuming the necessity of rigorous, homogenous and constant statistical data for the best visibility of this sector, our last interest resides in contributing to facilitate the adoption of public policies sustained in the promotion of the social economy as a key element to face the economic crisis in the Basque Country.
\end{abstract}

\title{
Keywords:
}

Social Economy, Economic Crisis, Measurement of Social Economy, Visibility of Social Economy, Public Policies.

\section{Claves ECONLIT}

P130, L310, C810, H120

\section{Introducción}

El presente artículo nace al amparo del intenso trabajo desarrollado desde el Observatorio Vasco de Economía Social (OVES/GEEB) en el marco de la elaboración del informe «Economía Social Vasca y Crisis Económica: análisis de su evolución socioeconómica entre 2009 y 2013" (Bretos et al., 2015) ${ }^{1}$, así como de la experiencia adquirida con la realización de anteriores informes sobre la situación de la Economía Social vasca que el OVES/GEEB lleva realizando anualmente desde el ańo 2009. A raíz de la investigación desarrollada desde este Observatorio, vienen advirtiéndose diversas dificultades y complejidades existentes a la hora de obtener información rigurosa, completa, homogénea y constante sobre el conjunto de entidades de la Economía Social vasca, lo que en última instancia obstaculiza la mejor visibilización de este sector tanto a nivel institucional como social y académico en la Comunidad Autónoma Vasca. Este escenario es especialmente perjudicial si tenemos en cuenta que, tal y como

1 Este trabajo está actualmente pendiente de publicación, previéndose que salga a la luz próximamente. 
arrojan los resultados de las mencionadas investigaciones, las entidades de la Economía Social vasca han soportado la crisis en mejores condiciones que las fórmulas empresariales convencionales, contribuyendo así al interés general gracias a la anteposición de la persona por encima de los intereses del capital.

No sería desmedido afirmar que en este contexto de crisis, un marco de políticas públicas sólidas dirigidas a la promoción y desarrollo de la Economía Social podría permitir una salida más efectiva, justa y equitativa de la crisis para el conjunto social. Por tanto, parece todavía más necesario que existan estadísticas rigurosas, homogéneas y continuadas sobre las entidades de Economía Social vascas que sustenten investigaciones empíricas acerca del análisis de cómo se comporta este sector. Ello, lógicamente, favorecería que desde las instituciones públicas se tuviese un conocimiento más generalizado para tomar medidas orientadas a hacer frente a la crisis mediante la potenciación de la Economía Social y, de forma general, fomentaría que en el conjunto de la sociedad se tuviese una mayor percepción de esta realidad.

En esta línea por tanto, el objetivo principal y aportación central de este artículo es identificar las dificultades concretas existentes en la Comunidad Autónoma Vasca para la obtención de datos socioeconómicos oficiales sobre el conjunto de entidades de Economía Social. A su vez, se presentan propuestas para superar estos obstáculos en aras de la mejor visibilización social e institucional de este sector a través de la correcta medición y valoración de sus entidades, el empleo que aglutinan y su contribución económica y social, que derive en última instancia en favorecer la adopción de políticas públicas dirigidas a fomentar la Economía Social y en el reconocimiento de sus potencialidades en términos sociales más amplios.

Para ello, se desarrolla el artículo de la siguiente manera. Tras esta introducción, el siguiente apartado se dedica al análisis y revisión en torno a la conceptualización y delimitación de la Economía Social en España y a la labor realizada por CIRIEC-España en el ámbito de la medición de la Economía Social, como referencia estatal en este campo. En el tercer apartado, se pone en valor el papel que juega el Observatorio Vasco de Economía Social (OVES/GEEB) a través de su origen, las actividades que desarrolla y su contribución a la Economía Social en el País Vasco. En el cuarto apartado, en aras de demostrar la necesidad de visibilización de la Economía Social, se exponen los principales resultados de los últimos informes del OVES/GEEB sobre el comportamiento de la Economía Social vasca ante la crisis económica actual. En el quinto apartado se recoge detalladamente la problemática concreta que debe enfrentar el OVES/GEEB para obtener información socioeconómica de cada una de 
las familias de la Economía Social en el País Vasco, resaltando las medidas adoptadas y proponiendo soluciones para superar estos obstáculos. Finalmente, en el sexto apartado se destacan las principales conclusiones del artículo.

\section{Aproximaciones a la conceptualización, delimitación y medición de la Economía Social}

\subsection{La conceptualización y delimitación de la Economía Social}

Históricamente, la conceptualización de la Economía Social, así como la identificación y delimitación de las organizaciones que la conforman, han sido objeto de amplios debates enmarcados en diferentes corrientes (Monzón, 2006; Chaves, 1997, 1999). Igualmente, han surgido y existen diversos conceptos como Tercer Sector, Economía Solidaria, Organización No Lucrativa o Empresa Social que lejos de distinguirse claramente de la naturaleza de la Economía Social, son conceptos afines, que incluso se diluyen en sus fronteras y que sin duda coexisten con mucho más en común de lo que les diferencia (Pérez de Mendiguren, et al, 2009; Monzón, 2006).

Tradicionalmente, se ha considerado a las Cooperativas, Mutualidades y Asociaciones como actores principales de la Economía Social. Todas estas manifestaciones asociativas tienen un núcleo y origen histórico común, y es que surgen como una respuesta de las clases sociales más desprotegidas y desfavorecidas, que estructuradas en organizaciones de autoayuda, se unieron para hacer frente a las duras condiciones laborales y de vida surgidas del desarrollo del capitalismo industrial entre los siglos XVIII y XIx (Monzón, 2009; 1989). En los últimos decenios se han incorporado las Sociedades Laborales y más recientemente otras entidades como los Centros Especiales de Empleo o las Empresas de Inserción. En este sentido, la crisis económica de los 70, punto de inflexión determinante del «giro neoliberal» que deriva en la menor capacidad de los Estados nacionales para dinamizar la economía (Teeple, 1995), en el aumento de las desigualdades (Galbraith, 2012; Stiglitz, 2012; Piketty, 2014), incremento del desempleo (Campbell y Pedersen, 2001; Harvey, 2005) y empobrecimiento de las condiciones socio-laborales (Kalleberg, 2009; Beck, 2000), supone también un impulso para la autogestión, el autoempleo y el estrechamiento de los lazos de solidaridad entre actores sociales y económicos. De esta manera, surgen diversas tipologías de organizaciones enmarcadas en diferentes formas jurídicas que, si bien no se encuadran en las formas clásicas de la Economía Social, sí guardan un es- 
trecho vínculo con su naturaleza social y democrática (Pérez de Mendiguren, Etxezarreta y Guridi, 2009). Así, en el último cuarto del siglo xx, el sector de la Economía Social experimenta un extraordinario impulso (Chaves, 2007; Monzón, 2009).

Desde un punto de vista científico-académico, la definición elaborada por Chaves y Monzón (2008) a propuesta del Comité Económico y Social Europeo (CESE) está ampliamente aceptada y es especialmente útil para comprender la naturaleza de las entidades que conforman este sector. Para los autores, «la Economía Social es un conjunto de empresas privadas organizadas formalmente, con autonomía de decisión y libertad de adhesión, creadas para satisfacer las necesidades de sus socios a través del mercado, produciendo bienes y servicios, asegurando o financiando, y en las que la eventual distribución entre los socios de beneficios o excedentes asi como la toma de decisiones no están ligadas directamente con el capital o cotizaciones aportados por cada socio, correspondiendo un voto a cada uno de ellos, o se llevan a cabo en todo caso mediante procesos democráticos y participativos de toma de decisiones. La economía social también agrupa a aquellas organizaciones privadas organizadas formalmente con autonomia de decisión y libertad de adhesión que producen servicios no de mercado a favor de las familias, cuyos excedentes, si los hubiera, no pueden apropiarse los agentes económicos que los crean, los controlan $o$ los financian".

En nuestro caso, en el año 2011 se produce un hecho especialmente significativo; la aprobación en España de la Ley 5/2011, de 29 de marzo, de Economía Social, que es capaz de integrar las nuevas realidades y experiencias de la Economía Social que se han adherido a las entidades tradicionales del sector (Etxezarreta y Morandeira, 2012). Aun con sus imperfecciones y carencias (Paniagua, 2011; Paz Canalejo, 2012) y el incumplimiento sistemático en la aplicación de sus contenidos por parte de la Administración Pública (Pérez de Uralde, 2014, 2012), es obvio que supone al menos la respuesta institucionalizada fuera del ámbito académico al reto de la conceptualización de la Economía Social y de la delimitación de las entidades que conforman este sector (Chaves, et al., 2013). Ello supone la superación de una primera dificultad a la que se debía hacer frente en el ámbito de la medición de la Economía Social, tanto a nivel de número de empresas, del empleo que aglutinan, y de su contribución económica: el previo consenso sobre la configuración de las entidades que conforman el sector.

En este sentido, el artículo 4 de la citada Ley 5/2011 define una serie de principios orientadores que se erigen como señas identitarias en torno a la conceptualización de la Economía Social, y el artículo 5 recoge de manera abierta e inclusiva las formas jurídicas y empresariales concretas que enarbolan el sector. 
"Artículo 4. Principios orientadores.

Las entidades de la economía social actúan en base a los siguientes principios orientadores:

a) Primacia de las personas y del fin social sobre el capital, que se concreta en una gestión autónoma y transparente, democrática y participativa, que lleva a priorizar la toma de decisiones más en función de las personas y sus aportaciones de trabajo y servicios prestados a la entidad o en función del fin social, que en relación a sus aportaciones al capital social.

b) Aplicación de los resultados obtenidos de la actividad económica principalmente en función del trabajo aportado y servicio o actividad realizada por las socias y socios o por sus miembros y, en su caso, al fin social objeto de la entidad.

c) Promoción de la solidaridad interna y con la sociedad que favorezca el compromiso con el desarrollo local, la igualdad de oportunidades entre hombres y mujeres, la cohesión social, la inserción de personas en riesgo de exclusión social, la generación de empleo estable y de calidad, la conciliación de la vida personal, familiar y laboral y la sostenibilidad.

d) Independencia respecto a los poderes públicos.

Artículo 5. Entidades de la economía social.

1. Forman parte de la economía social las cooperativas, las mutualidades, las fundaciones y las asociaciones que lleven a cabo actividad económica, las sociedades laborales, las empresas de inserción, los centros especiales de empleo, las cofradias de pescadores, las sociedades agrarias de transformación $y$ las entidades singulares creadas por normas especificas que se rijan por los principios establecidos en el artículo anterior.

2. Asimismo, podrán formar parte de la economía social aquellas entidades que realicen actividad económica y empresarial, cuyas reglas de funcionamiento respondan a los principios enumerados en el articulo anterior, $y$ que sean incluidas en el catálogo de entidades establecido en el artículo 6 de esta Ley.

3. En todo caso, las entidades de la economía social se regularán por sus normas sustantivas especificas.»

Conviene señalar que el OVES/GEEB asume la definición realizada por CIRIEC-Espańa, de la que GEZKI es miembro, pero la matiza y adapta en base a las especificaciones del País Vasco y a la Ley de Economía Social del año 2011. Así, desde el OVES/GEEB se propone el siguiente mapa que recoge las distintas tipologías de entidades que conforman el ecosistema de la Economía Social vasca. 


\section{Cuadro 1}

El Universo de la Economía Social desde el OVES-GEEB

\begin{tabular}{|c|c|c|}
\hline \multicolumn{3}{|c|}{ El universo de la Economía Social vasca } \\
\hline \multirow[t]{2}{*}{$\begin{array}{l}\text { Subsector } \\
\text { Productores de } \\
\text { Mercado }\end{array}$} & $\begin{array}{c}\text { Sociedades } \\
\text { no financieras }\end{array}$ & $\begin{array}{l}\text { - Cooperativas. } \\
\text { - Sociedades laborales. } \\
\text { - Centros especiales de empleo. } \\
\text { - Empresas de inserción. } \\
\text { - Cofradía de Pescadores. } \\
\text { - Sociedades Agrarias de Transformación. } \\
\text { - Otros productores privados de mercado (aso- } \\
\text { ciaciones, fundaciones y sociedades). }\end{array}$ \\
\hline & $\begin{array}{l}\text { Sociedades } \\
\text { financieras }\end{array}$ & $\begin{array}{l}\text { - Cooperativas de crédito. } \\
\text { — Entidades de previsión social voluntarias. } \\
\text { — Sociedades de garantía recíproca de entidades } \\
\text { de Economía Social. }\end{array}$ \\
\hline $\begin{array}{l}\text { Subsector } \\
\text { Productores de } \\
\text { no Mercado }\end{array}$ & $\begin{array}{l}\text { - Asociacione } \\
\text { - Fundacione } \\
\text { - Otras institu }\end{array}$ & $\begin{array}{l}\text { s de acción social. } \\
\text { s de acción social. } \\
\text { uciones sin fines de lucro al servicio de los hogares. }\end{array}$ \\
\hline
\end{tabular}

Fuente: OVES/GEEB.

\subsection{La labor de CIRIEC-España en el ámbito de la medición de la Economía Social en España}

Como se señala en su propia página web ${ }^{2}$, CIRIEC (Centro Internacional de Investigación e Información sobre la Economía Pública, Social y Cooperativa) es una organización científica internacional no gubernamental, cuyos objetivos son promover la búsqueda de información, la investigación científica y la difusión de trabajos sobre los sectores y actividades que tienen por principal finalidad la de servir al interés general: la acción de los poderes públicos en materia económica; los servicios públicos, las empresas públicas y las entidades de Economía Social. Su acción persigue, a este respecto, una información y un enriquecimiento mutuo de los profesionales y de los científicos. CIRIEC-España, sección nacional de CIRIEC en nuestro país, se constituyó como asociación en el año 1986, y su sede se encuentra en la Universidad de Valencia.

\footnotetext{
2 www.ciriec.es
} 
CIRIEC-España es sin duda la referencia estatal en el ámbito de la investigación de la Economía Social, habiendo desarrollado importantes estudios dedicados a la conceptualización, delimitación y medición de las entidades que forman parte del sector, tanto a nivel internacional como estatal. En este sentido, destaca por ejemplo la confección del «Informe para la Elaboración de una Ley de Fomento de la Economía Social» (Monzón, 2009), realizado en parte por el Grupo de Expertos de CIRIEC-España, que posteriormente se formalizaría en el borrador de anteproyecto de Ley de Economía Social presentado por el Ministerio de Trabajo e Inmigración.

En el campo de la medición del sector de la Economía Social han llevado a cabo importantes trabajos a nivel internacional dedicados a la delimitación y cuantificación de las entidades y el empleo que aglutina el sector de la Economía Social en la Unión Europea. Destaca por ejemplo el reciente estudio «La Economía Social en la Unión Europea» (Chaves y Monzón, 2012). A nivel exclusivamente estatal, se han desarrollado investigaciones similares de suma relevancia como el «Libro Blanco de la Economía Social en España» (Barea y Monzón, 1992), «La Economía Social en España en el año 2000» (Barea y Monzón, 2002), «La Economía Social en España en el año 2008: Ámbito, Magnitudes, Actividades y Tendencias» (Monzón, 2010), o el más reciente «Informe sobre las Grandes Cifras de la Economía Social en España: periodo 2011-2012» (Monzón, 2013).

Sobresale sin duda la elaboración de las cuentas satélite de la Economía Social, especialmente mediante el trabajo «Las Cuentas Satélite de las Cooperativas, Mutuas y Mutualidades de Previsión Social en España. Año 2008» (Barea y Monzón, 2011), elaborado junto con el Instituto Nacional de Estadística (INE).

El principal objetivo de esta investigación consistía en obtener información sobre las principales magnitudes económicas y otros indicadores de actividad no monetarios de dos grandes grupos de empresas de la Economía Social con un comportamiento homogéneo; las cooperativas y las mutuas y mutualidades. En tanto que en las Cuentas Nacionales no aparece desagregada la actividad de dichas empresas de la Economía Social (ya que viene agrupada en los sectores institucionales de sociedades no financieras e instituciones financieras), se revelaba necesario y fundamental elaborar cuentas satélite de cooperativas, mutuas y mutualidades. Esto es, la actividad económica llevada a cabo por las empresas de la Economía Social queda recogida en las Cuentas Nacionales en los sectores institucionales o ramas de actividad en los que se integran estas unidades, pero no se refleja de forma explícita, debido a las clasificaciones utilizadas por el Sistema Europeo de Cuentas Nacionales y Regio- 
nales (SEC-95). Por ello, la forma más adecuada que el SEC-95 recomienda para presentar los resultados contables de estas empresas es una cuenta satélite.

Estas cuentas satélite se elaboraron respetando los conceptos y clasificaciones básicos del cuadro central de la contabilidad nacional establecido por el Sistema Europeo de Cuentas Nacionales y Regionales vigente (SEC-1995), las Cuentas nacionales elaboradas por el Instituto Nacional de Estadística de España (INE) y la metodología del «Manual para la Elaboración de las Cuentas Satélite de las Empresas de la Economía Social: Cooperativas y Mutuas» (Barea y Monzón, 2007), de la Comisión Europea.

La elaboración de las cuentas satélite de la Economía Social ha supuesto, sin lugar a dudas, un paso extraordinario para dar una mayor visibilidad social, institucional y académica al sector, ya que estas cuentas han permitido reconocer la importancia económica y el valor añadido social de cooperativas, mutuas y mutualidades.

La realización de los distintos estudios e informes elaborados por CIRIEC-España se han podido desarrollar gracias a que han contado con la colaboración y el trabajo conjunto de diversos investigadores y expertos sectoriales, miembros de federaciones representativas del sector de la Economía Social y sus entidades, diferentes instancias de las Administraciones Pública, así como otros organismos. Cabe resaltar en este sentido, como ya señalábamos, la colaboración con el Instituto Nacional de Estadística (INE) para la elaboración de las cuentas satélite.

\section{El Observatorio Vasco de Economía Social (OVES/GEEB)}

No podemos contemplar la existencia del Observatorio Vasco de Economía Social (OVES/GEEB) sin poner en valor previamente la historia del Instituto de Derecho Cooperativo y Economía Social (GEZKI) de la Universidad del País Vasco (UPV/EHU). El Instituto GEZKI nació en 1987 en el seno de la Universidad del País Vasco (UPV/EHU) a partir de un grupo de profesores de la Facultad de Derecho. Su idea original consistió en atender a la falta de representatividad de la Economía Social en los planes académicos, de investigación y de formación en el ámbito de la Universidad Pública, a pesar de que la Economía Social ha tenido históricamente una fuerte presencia y enraizamiento en el contexto socioeconómico vasco. Surgía de esta manera el primer Instituto universitario público en el Estado dedicado específicamente a la Economía Social, convirtiéndose con 
los años en un referente en la visibilización de este sector (Pérez de Uralde, 2004).

Por su parte, el Observatorio Vasco de Economía Social (OVES/ GEEB) nace al amparo del Convenio de Colaboración del 30 de diciembre de 2008 firmado entre el Gobierno Vasco y la UPV/EHU, representada por el Instituto de Derecho Cooperativo y Economía Social (GEZKI), para la implantación progresiva de dicho Observatorio Vasco de Economía Social. De esta manera se daba respuesta a las resoluciones del Parlamento Vasco recogidas en el BOPV n. ${ }^{\circ}$ 153, de 25 de abril de 2008, sobre la necesidad de constituir una entidad de estas características.

El OVES/GEEB nació así con la finalidad de fomentar el desarrollo de la Economía Social mediante la identificación y cuantificación de su actividad en la Comunidad Autónoma del País Vasco, entendiendo ésta como el sector que aglutina a aquellas entidades de naturaleza privada cuya característica común es que se constituyen para satisfacer necesidades sociales y no para retribuir a inversores capitalistas, atendiendo a los principios y requisitos que posteriormente se establecerían por la Ley 5/2011 de Economía Social.

Desde su comienzo, el OVES/GEEB recibió el apoyo de los principales agentes de la Economía Social Vasca, así como de las principales Universidades vascas y otras personas de reconocido prestigio vinculadas a la Economía Social.

Destacan por otra parte los convenios de colaboración firmados por este Observatorio con el Observatorio Español de Economía Social de CIRIEC-España y con la Fundación Divina Pastora. Estos convenios son indicativo de la labor y esfuerzo realizado por el OVES/GEEB en aras de consolidarse como entidad de referencia con respecto a la Economía Social vasca. También, se han firmado acuerdos de colaboración con el Observatorio Iberoamericano de Empleo y la Economía Social (OIBESCOOP) y el Observatorio Portugués de Economía Social.

Los contenidos o áreas funcionales que se abordan desde el OVES/ GEEB son principalmente tres:

-Área socio-económica: captación y publicación de datos económicos y socio-laborales de las entidades de Economía Social, y su entorno socio-económico: Empleo, Entidades y datos económicos.

-Área jurídico-política: identificación tanto de la legislación y normativa general vigente y aplicable a la Economía Social Vasca como de las Políticas Públicas dirigidas a la Economía Social Vasca. 
-Área científico-académica: realización de publicaciones, informes, cursos, etc., con la colaboración de GEZKI y los otros institutos universitarios.

La actividad del OVES/GEEB consiste principalmente en la elaboración de informes anuales sobre la situación de la Economía Social vasca y en la edición de un boletín mensual ${ }^{3}$ de amplia difusión en el que se recogen las principales noticias relacionadas con la Economía Social vasca. Los informes anuales ${ }^{4}$ realizados desde este Observatorio tienen como finalidad responder al requerimiento hecho desde la Dirección de Economía Social del Gobierno Vasco de cara a conocer la situación actual de la Economía Social Vasca, incluyendo un análisis de la situación económica y socio-laboral, atendiendo a la Proposición no de Ley 36/2009 (Boletín Oficial del Parlamento Vasco n. ${ }^{\circ} 31$, del 30/10/2009) por la que el Parlamento Vasco instaba al Departamento de Empleo y Asuntos Sociales a que se elaborase un informe que analizase la situación del sector Cooperativo y de las demás entidades de la Economía Social.

El trabajo más completo realizado por el Observatorio se viene elaborando actualmente a raíz de la experiencia adquirida durante sus cinco años de vida. En dicho trabajo, "Economía Social Vasca y Crisis Económica: análisis de su evolución socioeconómica entre 2009 y 2013" (Bretos et al., 2015) se trata de ofrecer una visión lo más completa posible de la realidad de la Economía Social vasca y su comportamiento frente a la crisis económica a partir de datos estadísticos rigurosos y fiables, dotando de un carácter cualitativo al estudio mediante la interpretación de las tendencias experimentadas a nivel de empleo, número de entidades y datos económicos.

\section{Economía Social y crisis económica: resultados en el País Vasco}

Es bien conocido que históricamente las entidades de la Economía Social y especialmente las cooperativas, por lo general, han soportado mejor las crisis que otras estructuras empresariales tradicionales (Birchall, 2013), debido a su carácter contracíclico (Cornforth y Thomas, 1995;

3 Dicho Boletín puede consultarse en la página web del OVES/GEEB (www.oves-geeb. com).

${ }^{4}$ Desde el nacimiento del OVES/GEEB hasta el presente se han realizado Informes anuales sobre la situación de la Economía Social vasca para los ańos 2009, 2010, 2011, 2012 y 2013. Están disponibles igualmente en la página web del OVES/GEEB. 
Spear, 1996; Bartlett y Pridham, 1991; Westerdahl y Westlund, 1998; Grávalos y Pomares, 2001), logrando mantener en mejores condiciones el empleo, la calidad de las condiciones laborales y contribuyendo económicamente al Producto Interior Bruto de los países (Díaz y Marcuello, 2010), bajo la premisa de situar a la persona por encima de los intereses del capital.

En este sentido, las empresas de la Economía Social favorecen la distribución más igualitaria de la renta y riqueza, son capaces de corregir los desequilibrios del mercado de trabajo (paro, inestabilidad en el empleo y exclusión socio-laboral de los parados), potencian el desarrollo económico endógeno y construyen autonomía en los territorios, factores todos ellos fundamentales especialmente en coyunturas económicas adversas como la recesión padecida (Chaves, et al, 1999). En definitiva, ello demuestra que el fomento del ámbito empresarial de la Economía Social debe ser asumido por las Administraciones Públicas como un elemento clave de política económica con el que hacer frente a situaciones de crisis económica (Díaz y Marcuello, 2010; Chaves, 2007).

De la elaboración de los informes anuales realizados por el OVES/ GEEB sobre la situación de la Economía Social vasca (Morandeira, Bretos y Pérez de Uralde, 2014; Morandeira y Pérez de Uralde, 2013; 2012; 2011; 2010), así como del citado estudio "Economía Social Vasca y Crisis Económica: análisis de su evolución socioeconómica entre 2009 y 2013" (Bretos, et al, 2015) se desprenden unos resultados para la Economía Social en el País Vasco, los cuales exponemos a continuación, que son coincidentes con lo que señalan investigaciones similares como las referenciadas anteriormente.

En primer lugar, presentamos los datos sobre empleo y número de entidades de las cuatro grandes familias de la Economía Social ${ }^{5}$ (cuadros 2 y 3). Por otra parte, presentamos estos mismos datos (número de entidades y empleo), así como los datos relativos a la contribución económica (medido en el Valor Añadido Bruto generado), para el conjunto de entidades de la Economía Social vasca en los periodos bianuales 2008-2010 y 20102012, ya que son para los que se cuenta con la información completa para el conjunto del sector (cuadros 4, 5 y 6).

\footnotetext{
5 Éstas son Cooperativas, Sociedades Laborales, Centros Especiales de Empleo y Empresas de Inserción.
} 


\section{Cuadro 2}

Número agregado de empleos en el País Vasco de Cooperativas, Sociedades Laborales, Centros Especiales de Empleo y Empresas de Inserción

\begin{tabular}{lccccc}
\hline & 2009 & 2010 & 2011 & 2012 & 2013 \\
\hline Total CAV & 952.510 & 906.044 & 890.093 & 873.121 & 855.093 \\
$\quad$ Variación & - & $-4,88 \%$ & $-1,76 \%$ & $-1,91 \%$ & $-2,06 \%$ \\
\hline ECONOMÍA SOCIAL & 73.595 & 72.837 & 72.677 & 71.063 & 70.662 \\
$\quad$ Variación & - & $-1,03 \%$ & $-0,22 \%$ & $-2,22 \%$ & $-0,56 \%$ \\
\hline Participación & $7,73 \%$ & $8,04 \%$ & $8,17 \%$ & $8,14 \%$ & $8,26 \%$ \\
\hline
\end{tabular}

CAV: Comunidad Autónoma Vasca.

Fuente: Informes ańos 2009, 2010, 2011, 2012 y 2013 del OVES/GEEB sobre la «Situación de la Economía Social Vasca» e Informe «Economía Social Vasca y Crisis Económica: análisis de su evolución socioeconómica entre 2009 y 2013».

A raíz de estos resultados, es obvio que la Economía Social vasca no ha sido ajena a la difícil coyuntura económica que venimos atravesando desde 2008, sufriendo en mayor o menor medida sus consecuencias en el empleo, número de entidades y datos económicos.

\section{Cuadro 3}

Número agregado de entidades en el País Vasco de Cooperativas, Sociedades Laborales, Centros Especiales de Empleo y Empresas de Inserción

\begin{tabular}{lccccc}
\hline & 2009 & 2010 & 2011 & 2012 & 2013 \\
\hline Total CAV & 178.062 & 167.270 & 161.658 & 161.626 & 157.405 \\
Variación & - & $-6,06 \%$ & $-3,36 \%$ & $-0,02 \%$ & $-2,61 \%$ \\
\hline ECONOMÍA SOCIAL & 2.389 & 2.360 & 2.336 & 2.296 & 2.267 \\
$\quad$ Variación & - & $-1,21 \%$ & $-1,02 \%$ & $-1,71 \%$ & $-1,26 \%$ \\
\hline Participación & $1,34 \%$ & $1,41 \%$ & $1,45 \%$ & $1,42 \%$ & $1,44 \%$ \\
\hline
\end{tabular}

CAV: Comunidad Autónoma Vasca.

Fuente: Informes años 2009, 2010, 2011, 2012 y 2013 del OVES/GEEB sobre la «Situación de la Economía Social Vasca» e Informe «Economía Social Vasca y Crisis Económica: análisis de su evolución socioeconómica entre 2009 y 2013». 


\section{Cuadro 4}

Número agregado de empleos en el País Vasco

para el conjunto de entidades del sector de la Economía Social

\begin{tabular}{lcc}
\hline & $\mathbf{2 0 0 8 - 2 0 1 0}$ & $\mathbf{2 0 1 0 - 2 0 1 2}$ \\
\hline Total CAV & 906.044 & 873.121 \\
ECONOMÍA SOCIAL & 85.606 & 86.909 \\
Participación & $9,45 \%$ & $9,95 \%$ \\
\hline
\end{tabular}

CAV: Comunidad Autónoma Vasca.

Fuente: Informes años 2009, 2010, 2011, 2012 y 2013 del OVES/GEEB sobre la «Situación de la Economía Social Vasca» e Informe «Economía Social Vasca y Crisis Económica: análisis de su evolución socioeconómica entre 2009 y 2013».

\section{Cuadro 5}

Número agregado de entidades

para el conjunto del sector de la Economía Social en el País Vasco

\begin{tabular}{lrc}
\hline & $\mathbf{2 0 0 8 - 2 0 1 0}$ & $\mathbf{2 0 1 0 - 2 0 1 2}$ \\
\hline Total CAV & 167.270 & 161.626 \\
ECONOMÍA SOCIAL & 22.876 & 24.364 \\
Participación & $13,68 \%$ & $15,07 \%$ \\
\hline
\end{tabular}

CAV: Comunidad Autónoma Vasca.

Fuente: Informes años 2009, 2010, 2011, 2012 y 2013 del OVES/GEEB sobre la «Situación de la Economía Social Vasca» e Informe «Economía Social Vasca y Crisis Económica: análisis de su evolución socioeconómica entre 2009 y 2013».

\section{Cuadro 6}

Valor Ańadido Bruto (miles de euros)

generado por el conjunto del sector de la Economía Social en el País Vasco

\begin{tabular}{lcc}
\hline & $\mathbf{2 0 0 8 - 2 0 1 0}$ & $\mathbf{2 0 1 0 - 2 0 1 2}$ \\
\hline Total CAV & 61.304 .826 & 60.875 .938 \\
ECONOMÍA SOCIAL & 3.507 .264 & 3.300 .724 \\
Participación & $5,72 \%$ & $5,42 \%$ \\
\hline
\end{tabular}

CAV: Comunidad Autónoma Vasca.

Fuente: Informes años 2009, 2010, 2011, 2012 y 2013 del OVES/GEEB sobre la «Situación de la Economía Social Vasca» e Informe «Economía Social Vasca y Crisis Económica: análisis de su evolución socioeconómica entre 2009 y 2013». 
En este sentido, es apreciable que en los años 2009, 2010 y 2011, tanto a nivel de empleo como de número de entidades, la Economía Social vasca ha experimentado una ligera tendencia negativa, si bien es de destacar que, comparativamente con la economía convencional de la Comunidad Autónoma Vasca (CAV), ha sido capaz de mantener el número de empleos y entidades en mejores condiciones. Igualmente, es reseñable el significativo mantenimiento durante dichos ańos de la capacidad de generación de Valor Añadido Bruto de la Economía Social vasca.

Sin embargo, 2012 es el año en el que realmente la Economía Social vasca sufre de manera más dramática el impacto de la crisis económicafinanciera, experimentándose significativos descensos tanto en el empleo, como en el número de entidades y en la estabilidad económica. De esta manera, en el año 2012, la Economía Social vasca no fue capaz de resistir las consecuencias de la crisis en mejores condiciones que el conjunto de la economía convencional de la CAV. En cambio, en el año 2013 se amortigua la extraordinaria caída producida en 2012 tanto a nivel de empleo como de número de entidades. Si bien es cierto que no permite volver a alcanzar en términos generales las cifras previas de 2010 ó 2011, este año significa una cierta recuperación y además, por otra parte, la Economía Social vuelve a responder en mejores condiciones que el conjunto de la economía convencional de la CAV.

\section{Dificultades y propuestas desde el OVES/GEEB para la medición de la Economía Social vasca}

\subsection{Dificultades y obstáculos para la obtención de datos y la medición de la Economía Social en el País Vasco}

Atendiendo a la configuración asumida desde el OVES/GEEB sobre las entidades comprendidas en el sector de la Economía Social (véase el anterior cuadro 1), en la Comunidad Autónoma Vasca existe un primer gran obstáculo referente a la dispersión en diferentes instancias y departamentos del Gobierno Vasco, así como de sus organismos autónomos, de las funciones y responsabilidades sobre las distintas tipologías de entidades de Economía Social. Asimismo, se observa incluso que distintas instancias públicas asumen diversas actividades relacionadas con las diferentes tipologías de entidades de la Economía Social, como pueden ser funciones de registro administrativo, políticas de fomento, concesión de subvenciones...

Por ejemplo, en relación a Empresas de Inserción y Centros Especiales de Empleo, por un lado, la Dirección de Activación Laboral de LANBIDE define diferentes políticas para la inserción socio-laboral de colectivos de 

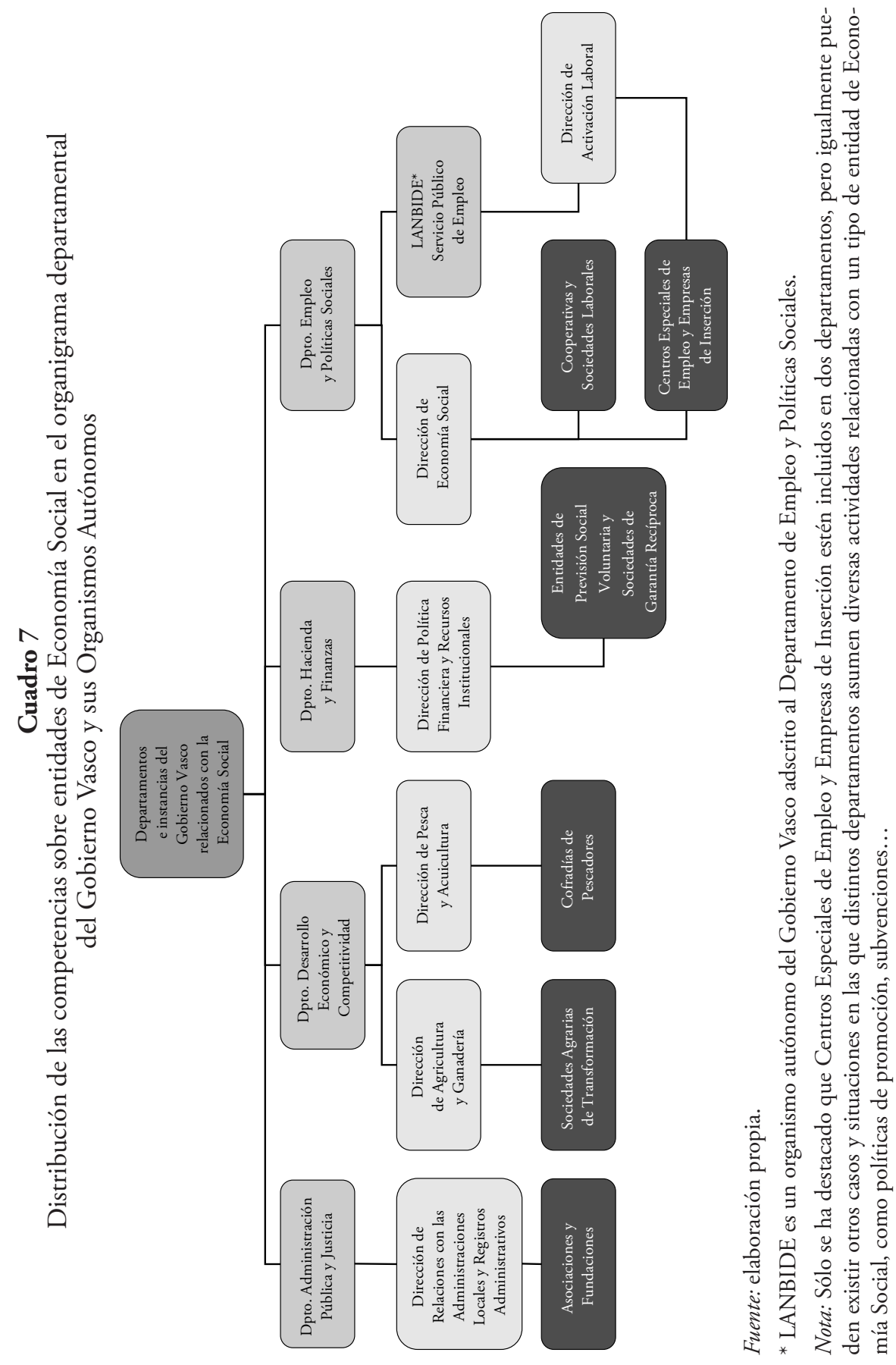

Revista Vasca de Economía Social • ISSN: 1698-7446 • e-ISSN: 2444-3107 GEZKI, n. ${ }^{\circ} 12,2015,7-34$ 
personas con discapacidad o en riesgo de exclusión y, por otro lado, la Dirección de Economía Social, integrada en el Departamento de Empleo y Asuntos Sociales, fomenta este tipo de entidades a través de medidas como las subvenciones a sus federaciones representativas, EHLABE y GIZATEA. Igualmente, en otros tipos de entidades de la Economía Social, es muy habitual que existan diferentes relaciones de responsabilidades y medidas desde diversas instancias públicas (véase el siguiente cuadro 7).

Este escenario, que no tiene que ser negativo en sí mismo, no facilita la ágil obtención de una información homogénea y análoga de carácter oficial para cada una de las tipologías de entidades de la Economía Social. Ante esta situación, desde el Observatorio Vasco de Economía Social se ha acudido a diversas fuentes de información para obtener datos socio-laborales y económicos rigurosos y fiables en aras de realizar un análisis lo más completo y estricto posible de la realidad de la Economía Social en el País Vasco. Entre las principales fuentes de información empleadas destacan los informes bianuales del Gobierno Vasco sobre la Economía Social, las estadísticas del Ministerio de Empleo y Seguridad Social, la información proporcionada por las tres Haciendas Forales, y los diferentes informes y datos obtenidos de las asociaciones representativas de las entidades.

Sin embargo, es de resaltar, por un lado, que los datos recogidos en los informes del Gobierno Vasco tienen una periodicidad bianual, lo que impide dotar de una continuidad anual a ciertos datos que se presentan en los informes del OVES/GEEB. Además, tradicionalmente, en los informes del Gobierno Vasco se analizaba exclusivamente a Cooperativas y Sociedades Laborales. Por el contrario, en su informe del año 2010, y reproduciendo la labor que ya se venía realizando previamente desde el OVES/ GEEB, comienzan a presentarse datos sobre el conjunto de entidades de la Economía Social en la configuración que posteriormente se recogería en la Ley 5/2011, de 29 de marzo, de Economía Social. De esta manera, dada su periodicidad bianual, los informes del Gobierno Vasco no recogen datos previos al año 2010 para las nuevas entidades definidas en la Ley. Es de destacar que desde el OVES/GEEB ya se venía recogiendo en sus anteriores informes el conjunto de entidades que posteriormente vendrían definidas en la Ley $5 / 2011$.

Por otra parte, es de subrayar que no existen entidades representativas o entes similares para todas las familias de la Economía Social, provocando que se disponga de una información mucho menor e imprecisa sobre las familias que no cuentan con entidad representativa que las aglutine. En el País Vasco existen entidades representativas o entes similares para Cooperativas (KonfeKoop-Confederación de Cooperativas de Euskadi), Sociedades Laborales (ASLE-Asociación de Sociedades Laborales de Euskadi), Centros Especiales de Empleo (EHLABE-Asociación de Entidades de Tra- 
bajo Protegido del País Vasco), Empresas de Inserción (GIZATEA-Asociación de Empresas de Inserción del País Vasco) y Entidades de Previsión Social Voluntaria (Federación de Entidades de Previsión Social Voluntaria de Euskadi). Recientemente, en mayo de 2015, se creó la Federación Vasca de Cofradías de Pescadores (El País, 2015).

A continuación se trata la problemática concreta para obtener información sobre cada una de las familias de la Economía Social en el País Vasco y las soluciones adoptadas desde el OVES/GEEB para disponer de los datos estadísticos correspondientes a nivel de número de entidades, empleo e información económica.

\section{CoOperativas y Sociedades Laborales}

La Dirección de Economía Social, integrada en el Departamento de Empleo y Políticas Sociales, es la encargada de dirigir y gestionar el Registro de Cooperativas de Euskadi. Sin embargo, desde el Observatorio se percibió que se llevaba una identificación actualizada y precisa de apenas el 60\% de las Cooperativas vascas. Respecto a las Sociedades Laborales, se encarga de controlar el cumplimiento de los requisitos establecidos en la Ley de Sociedades Laborales y otorgar la calificación o descalificación de «Sociedad Laboral» a las empresas.

Por tanto, ante la falta de información completa sobre estas entidades, desde el Observatorio se han empleado los datos sobre el número de entidades y empleos de Cooperativas y Sociedades Laborales proporcionados por el Ministerio de Empleo y Seguridad Social. A nivel exclusivamente de empleo, se han utilizado también los datos proporcionados por el Gobierno Vasco en los informes que elabora sobre la Economía Social ya que, si bien existe el problema de la periodicidad bianual empleada en éstos, por otra parte permiten realizar un análisis territorial y sectorial que no ofrece la información del Ministerio de Empleo y Seguridad Social.

Por otra parte, es de subrayar que las mismas referencias estadísticas, por ejemplo el número de empleos en Cooperativas del País Vasco, varían con diferentes cifras dependiendo de la fuente a la que se acuda, si los informes del Gobierno Vasco o el Ministerio de Empleo y Seguridad Social. Ante esta disfunción, desde el Observatorio se decidió utilizar los datos de los informes del Gobierno Vasco para realizar un análisis cualitativo de la estructura sectorial y territorial del empleo en el País Vasco, y los datos del Ministerio de Empleo y Seguridad Social para realizar un análisis cuantitativo de la evolución del empleo durante estos años.

A nivel de información económica, el Ministerio de Empleo y Seguridad Social sólo ofrece datos sobre la Cuenta de Pérdidas y Ganancias de las Cooperativas y Sociedades Laborales vascas hasta el año 2011, siendo ade- 
más los datos de este último año incompletos. Sin embargo, se ha podido obtener datos de las tres Haciendas Forales de Araba, Gipuzkoa y Bizkaia, aunque son referidos únicamente a Cooperativas. Los datos económicos relativos a las Sociedades Laborales se extraen nuevamente de los informes bianuales del Gobierno Vasco sobre la Economía Social.

\section{Centros Especiales de Empleo y Empresas de Inserción}

Por su parte, Centros Especiales de Empleo y Empresas de Inserción están incluidos en LANBIDE-Servicio Público de Empleo, un organismo autónomo del Gobierno Vasco. Sin embargo, no existen registros propiamente dichos, automatizados y actualizados, para ninguna de las dos. En cambio, ambos tipos de empresas están obligadas a presentar su memoria cuando piden las subvenciones anualmente, aunque las Empresas de Inserción por las características propias de las subvenciones no están obligadas a presentarla anualmente sino que normalmente lo hacen de manera bianual. Si bien desde el Observatorio se ha podido acceder a esta información, ésta no era completa para todos los años, sumando además las dificultades derivadas del estado de los datos, ya que estaban sin digitalizar (almacenados en formato papel).

Por tanto, la información pertinente se ha obtenido de las entidades representativas. Es de destacar en este sentido que dichas entidades, EHLABE y GIZATEA, no reúnen a todas y cada una de las empresas de su sector correspondiente, si bien ostentan un elevado grado de representatividad. Por un lado, la presencia de EHLABE dentro del sector de los Centros Especiales de Empleo en el País Vasco es bastante significativa, ya que sus entidades asociadas aglutinan algo más del $90 \%$ del conjunto del empleo especial de la Comunidad Autónoma Vasca. Por otro lado, destaca que las entidades representadas por GIZATEA suponen más del 95\% del total de Empresas de Inserción registradas en la Comunidad Autónoma Vasca.

\section{Cofradías de Pescadores y Sociedades Agrarias de Transformación}

En el Departamento de Desarrollo Económico y Competitividad están incluidas Cofradías de Pescadores y Sociedades Agrarias de Transformación, si bien el registro y control de las primeras depende de la Dirección de Pesca y Agricultura, y la gestión del registro de las segundas de la Dirección de Agricultura y Ganadería. Desde el Observatorio se ha solicitado formalmente el envío de los datos correspondientes sin haber obtenido respuesta alguna. Por tanto, nos hemos servido de la información disponible en los informes bianuales del Gobierno Vasco sobre la Economía Social. 
Sin embargo, la reciente creación de la nueva Federación Vasca de Cofradías de Pescadores podría facilitar la obtención futura de datos relativos a las cofradías vascas.

\section{AsOCIACIONES Y FUNDACIONES}

La Dirección de Relaciones con las Administraciones Locales y Registros Administrativos, integrada en el Departamento de Administración Pública y Justicia, lleva el registro de Asociaciones y Fundaciones, que es público en OpenData. Sin embargo, este registro no está actualizado y aunque contabiliza el total de Asociaciones y Fundaciones registradas en el País Vasco, muchas de ellas pueden haber dejado ya de funcionar o no realizar una actividad económica, por lo que la muestra puede estar sobredimensionada. Por otra parte, es de destacar que Eustat recogía anteriormente datos sobre las Asociaciones en el País Vasco, pero desde 2011 no ha actualizado estos datos. Por último, en relación a los datos que recogen los Informes bianuales del Gobierno Vasco sobre la Economía Social, cabe señalar que toman en cuenta exclusivamente a las Asociaciones de Utilidad Pública y Fundaciones, lo que no se corresponde con el total de Asociaciones y Fundaciones del sector de la Economía Social. Al respecto cabe señalar que la Ley 5/2011 de Economía Social señala que son entidades de Economía Social las Asociaciones y Fundaciones que desarrollan una actividad económica, respondiendo a los principios orientadores que se recogen en el artículo 4 de la Ley.

De esta manera, actualmente, no se dispone de ninguna fuente oficial que contabilice las Asociaciones y Fundaciones de manera adecuada a lo indicado en dicha Ley, ni existe una entidad representativa que aglutine al conjunto de Asociaciones o Fundaciones vascas de Economía Social. Por tanto, desde el Observatorio se han empleado las diversas fuentes de información mencionadas anteriormente para los diferentes años, en función de su disponibilidad y adecuación.

\section{Entidades de Previsión Social Voluntaria (EPSV)}

La Dirección de Política Financiera y Recursos Institucionales, integrada en el Departamento de Hacienda y Finanzas, se encarga entre otras funciones de gestionar el Registro de las EPSV así como de supervisar la documentación económica-financiera que estas entidades deben remitir a

${ }^{6}$ Se consideran Asociaciones de Utilidad Pública a las Asociaciones calificadas con la declaración de Utilidad Pública de acuerdo a los requisitos indicados en el artículo 32 de la Ley Orgánica 1/2002, de 22 de marzo, reguladora del Derecho de Asociación. 
la Administración de la Comunidad Autónoma. Es de señalar que desde el año 2004 se produjo una ralentización de las publicaciones periódicas de datos oficiales sobre dichas entidades, que anteriormente se obtenían de los informes elaborados por la Dirección de Trabajo y Seguridad Social del Departamento de Justicia, Empleo y Seguridad Social del Gobierno Vasco. Ante esta carencia, desde el Observatorio se comienzan a emplear las estadísticas e información proporcionada por la entidad representativa del sector, la Federación de Entidades de Previsión Social Voluntaria de Euskadi.

Por tanto, se emplean datos referidos únicamente a las entidades federadas, si bien sobresale su sólida representatividad dentro del total del sector de las EPSV de Euskadi. En este sentido, estimativamente, las EPSV federadas representaban casi el 50\% del total de las EPSV de Euskadi. Sin embargo, la práctica totalidad de las EPSV de las categorías Empleo, Asociados e Individuales, esto es, las más relevantes, estaban agrupadas en la Federación, quedando por tanto fuera la mayoría de EPSV enmarcadas en otras modalidades. Este dato queda reflejado en el hecho de que el patrimonio de las EPSV federadas representa algo más del $98 \%$ del total del patrimonio de todas las EPSV de Euskadi.

\section{Cooperativas de Crédito}

La información relativa a las Cooperativas de Crédito de Euskadi, que hasta 2012 eran dos, Caja Laboral Popular Sociedad Cooperativa de Crédito e Ipar-Kutxa Rural Sociedad Cooperativa de Crédito, se obtenían de sus Informes de Auditorias de Cuentas Anuales (Individuales). En 2012 ambas entidades se fusionan dando origen a la actual Laboral Kutxa. Los datos sobre dicha entidad se obtienen igualmente de sus Informes de Auditorias de Cuentas Anuales (Individuales).

\section{SOCIEDADES DE GARANTÍA RECÍPROCA (SGR)}

En primer lugar debemos señalar que las Sociedades de Garantía Recíproca propiamente dichas no están expresamente incluidas en la Ley 5/2011 de Economía Social y por lo tanto no se consideran en general parte del sector. En cualquier caso, bien es cierto que esta Ley es inclusiva y abierta, lo que permite la introducción de nuevas entidades que se consideren de la Economía Social.

En este sentido, desde el OVES/GEEB se ha considerado tradicionalmente que en el País Vasco existe una Sociedad de Garantía Recíproca que debe estar incluida en el sector de la Economía Social vasca, "Oinarri Sociedad de Garantía Recíproca para la Economía Social S.G.R.». Oinarri, 
que opera actualmente para todas la pymes del País Vasco, es la única SGR del Estado especializada en empresas de la Economía Social. En 1996 surgió con tal carácter de exclusividad para este sector, pero en 2001 abrió su ámbito de actuación a todas las empresas. Sin embargo, el 60\% de su actividad a día de hoy se desarrolla dentro de las empresas de la Economía Social siendo la única S.G.R. especializada en este campo y la mayor parte de sus empresas, un 92\%, tiene menos de 50 trabajadores. En este caso obtenemos los datos correspondientes para la elaboración del informe de la propia entidad Oinarri S.G.R. que hace públicos sus datos en sus memorias anuales.

Vista la problemática específica para la obtención de información sobre cada una de las entidades concretas de la Economía Social en el País Vasco, desde el Observatorio clasificamos estas dificultades en cuatro grandes grupos:

- Inexistencia de publicaciones periódicas de estadísticas oficiales para todas las entidades que conforman la Economía Social.

— La inaccesibilidad a datos oficiales de algunas de las entidades de Economía Social. Se han realizado contactos con diferentes instancias oficiales pero en algunos casos no se ha obtenido respuesta alguna.

—Dificultades derivadas del estado de los datos de algunos de los Registros Oficiales. Por ejemplo, datos almacenados en formato papel por lo que exige una recogida de datos manual de cada una de las entidades que supone un coste inasumible, por el momento, por parte del OVES/GEEB.

—Disparidad y divergencia de los mismos datos entre distintas fuentes y con diferentes metodologías.

Es obvio que las mencionadas dificultades para la obtención de información socioeconómica para toda la Economía Social vasca obstaculizan la mejor visualización de la realidad de este sector, y es que, la diversidad de fuentes, las diferencias en las variables que se trabajan y los distintos horizontes temporales tratados, no permiten realizar un análisis estricto y totalmente homogéneo para el conjunto de la Economía Social. Sin embargo, no hay lugar a dudas de que los resultados de los diferentes informes del OVES/GEEB, de los que hemos presentado los principales resultados entre 2009 y 2013, son una referencia solvente que se aproximan fehacientemente a la realidad del sector de la Economía Social vasca gracias a que los datos mostrados, si bien incompletos, son significativos y fiables. 


\subsection{Propuestas y soluciones desde el OVES/GEEB para la correcta medición de la Economía Social en el País Vasco}

Desde el OVES/GEEB entendemos interesante proponer ciertas cuestiones para la mejora de esta situación, ya que en el marco de la mejor visibilización social y reconocimiento institucional de la Economía Social es imprescindible que exista una correcta medición en términos de contabilización del número de entidades, del empleo que aglutinan y de los datos económicos, así como de su impacto social y económico.

Para ello, en primer lugar, consideramos que sería de especial utilidad que existiese un único departamento o instancia del Gobierno Vasco que centralizase los registros administrativos respectivos a todas las tipologías de entidades de la Economía Social vasca, y que absorbiese la totalidad de las competencias relacionadas con dichas entidades en su integridad, sin que ello suponga perjuicio para que cada Departamento o Dirección pueda proponer diversas políticas públicas para cada una de las tipologías de entidades que conforman el sector de la Economía Social en el País Vasco. En este sentido, parecería lógico que la Dirección de Economía Social, o en su defecto una Viceconsejería más amplia, llevase a cabo un redimensionamiento que le permitiese abarcar la totalidad del sector de la Economía Social. Ello obviamente facilitaría la comunicación con la Administración Pública y la extracción de los datos pertinentes para la medición precisa del sector de la Economía Social, así como también facilitaría un mayor desarrollo de políticas de fomento del sector.

Por otra parte, consideramos que el propio Observatorio Vasco de Economía Social (OVES/GEEB) puede situarse como una referencia para superar las barreras y disfunciones que plantean las mencionadas dificultades en aras de disponer de datos completos y homogéneos para el conjunto de entidades de la Economía Social vasca, afrontando para ello un trabajo estadístico periódico mediante una labor de recopilación y agregación de fuentes. Si bien el OVES/GEEB no es fuente de elaboración primaria de datos, en tanto que aquella es función de los organismos oficiales y las entidades del sector, sí puede erigirse como ente para el análisis y agregación de datos. En este sentido, el componente valorativo que integran sus estudios e investigaciones le otorga a éstos un valor ańadido propio derivado del carácter cualitativo y calificativo con que son tratados los datos sobre el sector de la Economía Social vasca.

En cualquier caso, para desarrollar este trabajo es necesaria la estrecha colaboración y el trabajo de todas las partes, desde las Administraciones Públicas, especialmente las Haciendas Forales, otros organismos oficiales relacionados con la elaboración y obtención de datos estadísticos, entre los que destacaría fundamentalmente el EUSTAT, hasta los propios actores 
involucrados en el sector de la Economía Social vasca como son las entidades y sus asociaciones representativas. Los últimos informes y estudios realizados por el OVES/GEEB sobre la evolución de la Economía Social vasca son una clara muestra de ello y tratan de avanzar en esta línea, desarrollando un trabajo que no encontramos en informes similares al presentar datos cruzados sobre distintas fuentes oficiales, y a su vez, ofreciendo datos solventes y representativos de distintas entidades de la Economía Social obtenidos de ellas mismas o sus asociaciones representativas.

\section{Conclusiones}

La falta de visibilidad social e institucional de la Economía Social se ha erigido históricamente como una grave carencia del sector, incluso en el País Vasco, una región caracterizada tradicionalmente por el fuerte arraigo social y económico de estas experiencias. En el ámbito académico se han dado pasos importantes en estos últimos años para visibilizar esta realidad con el firme compromiso de las universidades, destacando la creación del Máster en Economía Social y Solidaria por parte de la UPV/EHU y GEZKI, y del Máster en Economía Social y Empresa Cooperativa de Mondragon Unibertsitatea, o la labor del Instituto de Estudios Cooperativos de la Universidad de Deusto.

La entrada en vigor en España de la Ley 5/2011 de la Economía Social ha supuesto cuanto menos, desde una perspectiva institucional, cerrar el decimonónico debate sobre la conceptualización y delimitación del sector, suponiendo la mejor exteriorización y percepción de esta realidad. Ello ha permitido a su vez superar una primera dificultad para la medición de la Economía Social: el previo consenso sobre las entidades que configuran el sector. Sin embargo, a raíz del trabajo desarrollado desde el OVES/GEEB en el marco de la identificación, cuantificación, medición y valoración de las entidades de la Economía Social, se hace evidente que en el País Vasco existen dificultades significativas para la obtención de datos oficiales, rigurosos y fiables que permitan un seguimiento cuantitativo constante del sector de la Economía Social.

Sin duda, contar con estadísticas completas, homogéneas y constantes sobre el conjunto de entidades de la Economía Social vasca es una de las claves para abordar el reto de la mejor visibilización del sector. Esta necesidad se revela mucho más ineludible cuando observamos que, como se ha expuesto aquí, la Economía Social ha soportado en mejor condiciones que el sector empresarial convencional las consecuencias de la crisis económica que venimos padeciendo desde finales del año 2008. Pensamos en última instancia que la fácil accesibilidad a datos oficiales y constantes sobre 
la Economía Social estaría sustentando un mayor número de investigaciones en las que se analicen el comportamiento de la Economía Social en el País Vasco y sus extraordinarias contribuciones en términos sociales y económicos. Entendemos que también se fomentaría así la adopción de políticas públicas dirigidas a la potenciación y favorecimiento de la Economía Social, especialmente relevantes en la actualidad para lograr una salida más social y justa de la crisis económica.

En el presente artículo se trata de alertar sobre esta problemática, identificando las dificultades y disfunciones concretas para la obtención de estadísticas oficiales de la Economía Social vasca que obstaculizan la correcta y precisa medición del sector, con el objetivo último de facilitar la adopción de las medidas necesarias para superar estas barreras por parte de las instancias y organismos pertinentes. En este sentido, se propone que una sola viceconsejería centralice, a efectos estadísticos al menos, los datos relativos a todas las tipologías de entidades de la Economía Social vasca. Por otra parte, se sostiene que el OVES/GEEB puede situarse como una referencia para enfrentar la problemática relacionada con la publicación de datos estadísticos consistentes, fiables y homogéneos a través de una labor de agregación y cruce de datos. Para ello, será necesaria la estrecha colaboración de todas las partes implicadas, profundizando así la relación que ya se viene teniendo con entes públicos y entidades representativas del sector. En este ámbito, y siguiendo ejemplos como el de la colaboración entre CIRIECEspaña y el Instituto Nacional de Estadística (INE) para la elaboración de las cuentas satélite, podría ser especialmente fructífero marcos de relación y trabajo conjunto entre el OVES/GEEB y EUSTAT para la consolidación y visibilización del sector de la Economía Social en el País Vasco. De momento, entre los objetivos a corto plazo del OVES/GEEB se encuentra la elaboración de las cuentas satélite para las cooperativas en el País Vasco gracias a los datos obtenidos durante estos últimos años y al apoyo técnico de expertos del CIRIEC.

\section{Referencias bibliográficas}

Barea, J. y Monzón, J.L. (2011): Las Cuentas Satélite de las Cooperativas, Mutuas y Mutualidades de Previsión Social en España: Año 2008, CIRIEC-INE, Madrid.

Barea, J. y Monzón, J.L. (2002): La Economía Social en España en el año 2000, CIRIEC-España, Valencia.

Barea, J. y Monzón, J.L. (2007): Manual para la Elaboración de las Cuentas Satélite de las Empresas de la Economía Social: Cooperativas y Mutuas, CIRIEC-España, Bruselas. 
Barea, J. y Monzón, J.L. (1992): Libro Blanco de la Economía Social en España, Ministerio de Trabajo y Seguridad Social, Madrid.

Bartlett, W. y Pridham, G.S. (1991): "Cooperative Enterprises in Italy, Portugal and Spain: History, Development and Prospects», Journal of Interdisciplinary Economics, 4.

Beck, U. (2000): Un nuevo mundo feliz. La precariedad del Trabajo en la Era de la Globalización, Paidós, Barcelona.

Birchall, J. (2013): "The Potential of Cooperatives during the Current Recession: Theorizing Comparative Advantage», Journal of Entrepreneurial and Organization Diversity, 2 (1), 1-22.

Bretos, I.; Morandeira, J. y Pérez de Uralde, J.M. (2015): Economía Social Vasca y Crisis Económica: Análisis de su Evolución Socioeconómica entre 2009 y 2013, Observatorio Vasco de Economía Social (OVES/GEEB), Universidad del País Vasco (UPV/EHU), Donostia-San Sebastián (próxima publicación).

Campbell, J.L. y Pedersen, O.K. (2001): The Rise of Neoliberalism and Institutional Analysis, Princeton University Press, Princeton-Oxford.

Chaves, R. (2007) (Coord.): La Economía Social en las Politicas Públicas en España, Ministerio de Trabajo, Madrid.

Chaves, R. (1999): «La Economía Social como Enfoque Metodológico, como Objeto de Estudio y como Disciplina Científica», CIRIEC-España, Revista de Economía Pública, Socialy Cooperativa, 33, 115-139.

Chaves, R. (1997): «Economía Política de la Economía Social. Una revisión de la Literatura Económica Reciente», CIRIEC-España, Revista de Economía Pública, Social y Cooperativa, 25, 141-162.

Chaves, R. y Monzón, J.L. (2012): La Economía Social en la Unión Europea, Informe del Comité Económico y Social Europeo, Bruselas.

Chaves, R. y Monzón, J.L. (2008): La Economía Social en la Unión Europea, Informe del Comité Económico y Social Europeo, Bruselas.

Chaves, R.; Monzón, J.L.; Pérez de Uralde, J.M. y Radrigán, M. (2013): «La Economía Social en Clave Internacional. Cuantificación, Reconocimiento Institucional y Visibilidad Social en Europa, Iberoamérica y Norte de África», REVESCO, Revista de Estudios Cooperativos, 112.

Chaves, R. et al (1999): «La Economía Social y la Política económica», en JORDÁN, J.M. (ed.) (1999): Politica Económica y Actividad Empresarial, Valencia, Tirant lo Blanc.

Cornforth, C. y Thomas, A. (1995): "Les Cooperatives de Travail au Rouyaume Uni. Declin ou Croissance?», Revue de Etudes Cooperatives, Mutualistes et Associatives, 255 (53).

Díaz, M. y Marcuello, C. (2010): «Impacto Económico de las Cooperativas. La Generación de Empleo en las Sociedades Cooperativas y su Relación con el PIB», CIRIEC-España, Revista de Economía Pública, Social y Cooperativa, 67, 24-44.

EL PAÍS (2015): «Urkullu anuncia la creación de la Federación Vasca de Cofradías», El País, 8 de mayo de 2015. Disponible en: http://ccaa.elpais.com/ 
ccaa/2015/05/08/paisvasco/1431099030_706701.html (recuperado el 20 de mayo de 2015).

Etxezarreta, E. y Moranderia, J. (2012): «Consideraciones Conceptuales sobre la Economía Social a la luz de la Ley 5/2011», Revista Vasca de Economía Social, n. ${ }^{\circ}$, pp. 7-37.

Galbraith, J. (2012): Inequality and Instability. A Study of the World Economy Just Before the Great Crisis, New York: Oxford University Press.

Grávalos, M.A. y Pomares, I. (2001): «Cooperativas, Desempleo y Efecto Refugio", REVESCO, Revista de Estudios Cooperativos, 74, 69-84.

Harvey, D. (2005): A Brief History of Neoliberalism, Oxford University Press, Oxford-New York.

Kalleberg, A. (2009): «Precarious Work, Insecure Workers: Employment Relations in Transition", American Sociological Review, 74 (1), 1-22.

Monzón, J.L. (dir.) (2013): Informe sobre las Grandes Cifras de la Economía Social en España (periodo 2011-2012), CIRIEC-España, Valencia.

Monzón, J.L. (dir.) (2010): La Economía Social en España en el Año 2008: Ámbito, Magnitudes, Actividades y Tendencias, CIRIEC-España, Valencia.

Monzón, J.L. (coord.) (2009): Informe para la Elaboración de una Ley de Fomento de la Economía Social, CIRIEC-España, Valencia-Madrid.

Monzón, J.L. (2006): «Economía Social y Conceptos Afines: Fronteras Borrosas y Ambigüedades Conceptuales del Tercer Sector», CIRIEC-España, Revista de Economía Pública, Social y Cooperativa, 56, 9-24.

Monzón, J.L. (1989): Las cooperativas de Trabajo Asociado en la Literatura Económica y en los Hechos, Ministerio de Trabajo y Seguridad Social, Madrid.

Morandeira, J.; Bretos, I. y Pérez de Uralde, J.M. (2014): Informe de Situación de la Economía Social Vasca: Año 2013, Observatorio Vasco de Economía Social (OVES/GEEB), Universidad del País Vasco (UPV/EHU), DonostiaSan Sebastián.

Morandeira, J. y Pérez de Uralde, J.M. (2013): Informe de Situación de la Economía Social Vasca: Año 2012, Observatorio Vasco de Economía Social (OVES/GEEB), Universidad del País Vasco (UPV/EHU), Donostia-San Sebastián.

Morandeira, J. y Pérez de Uralde, J.M. (2012): Informe de Situación de la Economía Social Vasca: Año 2011, Observatorio Vasco de Economía Social (OVES/GEEB), Universidad del País Vasco (UPV/EHU), Donostia-San Sebastián.

Morandeira, J. y Pérez de Uralde, J.M. (2011): Informe de Situación de la Economía Social Vasca: Año 2010, Observatorio Vasco de Economía Social (OVES/GEEB), Universidad del País Vasco (UPV/EHU), Donostia-San Sebastián.

Morandeira, J. y Pérez de Uralde, J.M. (2010): Informe de Situación de la Economía Social Vasca: Año 2009, Observatorio Vasco de Economía Social (OVES/GEEB), Universidad del País Vasco (UPV/EHU), Donostia-San Sebastián. 
Paniagua, M. (2011): Las Empresas de la Economía Social. Más allá del Contenido de la Ley 5/2011 de Economía Social, Marcial Pons, Madrid.

Paz Canalejo, N. (2012): Comentario Sistemático a la Ley 5/2011, de 29 de marzo, de Economía Social, Tirant lo Blanch-Reformas, Valencia.

Pérez de Mendiguren, J.C.; Etxezarreta, E. y Guridi, L. (2009): «Economía Social, Empresa Social y Economía Solidaria: Diferentes Conceptos para un Mismo Debate». Papeles de Economía Solidaria, n. ${ }^{\circ}$ 1. REAS, Bilbao.

PÉrez de Uralde, J.M. (2014): «La Inaplicación de la Ley de Economía Social en un Contexto de Crisis Económica. Algunas Interpretaciones y Propuestas», CIRIEC-España, Revista de Economía Pública, Social y Cooperativa, 81, 33-59.

Pérez de Uralde, J.M. (2012): «Ley 5/2011, de 29 de marzo, de Economía Social: ¿su Aplicación es Cosa de Todos? (pregunta retórica)», Revista Deusto Estudios Cooperativos, 1, 79-92.

Pérez de Uralde, J.M. (2004): «GEZKI, una Intensa Historia en la Formación, Investigación y Promoción Universitaria de la Economía Social», Revista Vasca de Economia Social, n. ${ }^{\circ}$ 0, pp. 7-22.

Piketry, T. (2014): Capital in the 21st Century, Harvard University Press, Cambridge.

Spear, R. (1996): "Cooperativas y Empleo en el Reino Unido», CIRIEC-España, Revista de Economía Pública, Social y Cooperativa, 15, 25-31.

Stiglitz, J.E. (2012): El Precio de la Desigualdad: el 1\% de la Población Tiene lo que el 99\% Necesita, Taurus, Madrid.

Teeple, G. (1995): Globalization and the Decline of Social Reform, Toronto, Garamond.

Westerdahl, S. y Westlund, H. (1998): «Social Economy and New Jobs: a Summary of Twenty Case Studies on European Regions», Annals of Public and Cooperative Economics, 69:2, 193-218.

\section{Agradecimientos}

Quisiera agradecer sinceramente las sugerencias y propuestas recibidas por parte de José María Pérez de Uralde, Director del OVES/GEEB y Presidente de CIRIEC-España, y de Jon Morandeira Arcas, investigador y miembro del Instituto GEZKI y anterior técnico investigador del OVES/ GEEB. 